Epidemiology and Infection

cambridge.org/hyg

\section{Original Paper}

Cite this article: Korcinska MR, Dalsgaard Bjerre K, Dam Rasmussen L, Tvenstrup Jensen E, Fischer TK, Barrasa A, Ethelberg S (2020). Detection of norovirus infections in Denmark, 2011-2018. Epidemiology and Infection 148, e52, 1-7. https://doi.org/10.1017/ S0950268820000461

Received: 10 August 2019

Revised: 21 January 2020

Accepted: 29 January 2020

\section{Key words:}

Epidemiology; hospital-acquired (nosocomial) infections; norovirus; Norwalk agent and related viruses; surveillance

Author for correspondence:

S. Ethelberg, E-mail: SET@ssi.dk

\title{
Detection of norovirus infections in Denmark, 2011-2018
}

\author{
M. R. Korcinska ${ }^{1,2}$ (D) K. Dalsgaard Bjerre ${ }^{1}$, L. Dam Rasmussen 3 , \\ E. Tvenstrup Jensen ${ }^{1}$, T. K. Fischer ${ }^{4,5}$, A. Barrasa ${ }^{2,6}$ and S. Ethelberg ${ }^{1,7}$
}

${ }^{1}$ Department of Infectious Disease Epidemiology and Prevention, Statens Serum Institut, Copenhagen, Denmark; ${ }^{2}$ European Programme for Intervention Epidemiology Training (EPIET), European Centre for Disease Prevention and Control, Stockholm, Sweden; ${ }^{3}$ Department of Virus and Microbiological Special Diagnostics, Statens Serum Institut, Copenhagen, Denmark; ${ }^{4}$ Department of Research, Nordsjaellands Hospital, Hilleroed, Denmark;

${ }^{5}$ Departments of Infectious Diseases and Global Health, University of Southern Denmark, Odense, Denmark; ${ }^{6}$ National Centre for Epidemiology, Institute of Health Carlos III (ISCIII), Madrid, Spain and ${ }^{7}$ Department of Public Health, Global Health Section, University of Copenhagen, Copenhagen, Denmark

\begin{abstract}
Norovirus (NoV) infections occur very frequently yet are rarely diagnosed. In Denmark, NoV infections are not under surveillance. We aimed to collect and describe existing laboratorybased NoV data. National NoV laboratory data were collected for 2011-2018, including information on patient identification number, age and sex, requesting physician, analysis date and result. We defined positive patient-episodes by using a 30-day time window and performed descriptive and time series analysis. Diagnostic methods used were assessed through a survey. We identified 15809 patient-episodes (11\%) out of 142648 tested patients with an increasing trend, 9366 in 2011 vs. 32260 in 2018. This corresponded with a gradual introduction of polymerase chain reaction analysis in laboratories. The highest positivity rate was in patients aged $<5$ years $(15 \%)$ or $>85$ years $(17 \%)$. There was a large difference in test performance over five Danish geographical regions and a marked seasonal variation with peaks from December to February. This is the first analysis of national NoV laboratory data in Denmark. A future laboratory-based surveillance system may benefit public health measures by describing trend, burden and severity of seasons and possibly pinpoint hospital outbreaks.
\end{abstract}

(C) The Author(s), 2020. Published by Cambridge University Press. This is an Open Access article, distributed under the terms of the Creative Commons Attribution licence (http://creativecommons.org/licenses/by/4.0/), which permits unrestricted re-use, distribution, and reproduction in any medium, provided the original work is properly cited.

\section{CAMBRIDGE} UNIVERSITY PRESS

\section{Introduction}

Norovirus $(\mathrm{NoV})$ is the most common cause of acute gastroenteritis and outbreaks worldwide [1]. Recent systematic reviews estimate that NoV causes 18\% (95\% CI 17-20\%) of diarrhoeal disease worldwide $[1,2]$. The exemplar clinical case is an acute and self-limiting gastroenteritis that can be managed by rehydration therapy. The virus is highly contagious and transmitted from person-to-person often indirectly via food, water, hands or contaminated surfaces. NoV can easily spread via vomit or faecal contamination, especially in the absence of appropriate hygienic conditions. The majority of the reported outbreaks in developed countries take place in healthcare facilities, including nursing homes. These outbreaks often affect vulnerable people, such as hospitalised patients or the elderly. The outbreaks are disruptive and costly to health services and are best managed through infection control measures such as improved hygiene and patient isolation [3-9]. In Northern European countries, NoV infections commonly occur in the winter months and have been referred to as 'winter vomiting disease' [10].

Improved diagnostics have transformed the perception of NoV from a rarely laboratorydiagnosed infection in the early 90 s to the leading cause of gastroenteritis in people of all ages worldwide $[11,12]$. Over the last two decades, NoV has been increasingly diagnosed by enzyme-linked immunosorbent assay (ELISA) or reverse transcription-polymerase chain reaction (RT-PCR) assay. However, the surveillance of $\mathrm{NoV}$ is challenging, as most infectious episodes are not diagnosed. Patients may never consult healthcare professionals and even when medical care is sought, NoV testing will rarely be conducted due to the expected selflimiting course of the disease combined with the lack of therapeutic consequences $[6,13]$. There is limited information about the number of countries, where NoV is under surveillance, but for the reasons mentioned above, passive surveillance systems would likely be of limited value as early warning systems e.g. for hospital preparedness planning during excessive NoV seasons. However, a study of laboratory surveillance data from England and Wales has suggested a potential value of combining different information sources (information about the infection aetiology, seasonality, virology factors etc.) into a virus early warning system [14].

In Denmark, a country with 5.8 million inhabitants, NoV is thought to be a common infection. A recent burden of disease study estimated 185000 cases (incidence 3203 per 100000 inhabitants), 26 deaths and 485 disability-adjusted life years to have occurred in 2017 [15]. 
Moreover, NoV is a frequent cause of outbreaks in healthcare institutions, the most common aetiology among foodborne infections [16-20] and most frequent cause of foodborne outbreaks, responsible for $33 \%$ of known such outbreaks in 2018 [21]. $\mathrm{NoV}$ is currently not covered by national disease surveillance and there are no national guidelines or recommendations covering the practices at regional laboratories, thus there is no information on the laboratory practices. We took advantage of the recently implemented Danish Microbiology Database (MiBa) [22] to compile national diagnostic data from 2011 to 2018, in order to learn more about the epidemiology of the infections in Denmark. In addition, we conducted a survey to investigate the current laboratory testing practices.

\section{Methods}

\section{Data source}

In Denmark, hospital services, including microbiological analyses, are conducted within five geographic administrative regions. Diagnosis of NoV in Denmark is undertaken at publicly financed clinical microbiology laboratories (KMAs). Currently, there are 10 KMAs, which use different diagnostic methods and laboratory information systems. During the study period $12 \mathrm{KMAs}$ existed (including the reference laboratory) as some laboratories have recently been merged. In 2010, the MiBa was created, which contains copies of all electronic KMA analysis reports in Denmark, which can later be identified by three parameters: an MiBa identification number, the patient's unique civil registry number (CPR) and the report ID number used by the KMA. All Danish residents are registered by a unique population register (CPR) number [23], which allows for the elimination of duplicate recordings and for linking of data between registries. Although all KMAs provide information in coded form, a national standard for coding is not applied for all variables. Some elements in the reports follow national coding standards while other elements follow local standards. To extract data on NoV diagnostics from the $\mathrm{MiBa}$, we searched for all relevant codes and remapped them through a standardisation process. NoV laboratory data were compiled for the period of 2011-2018 including information on the requesting physician, requested analysis, results and person information. Information about Danish general and age-specific sizes of the population was obtained from Denmark Statistics [24]. As the laboratory diagnostic methods were changing over time, the study period was divided into two intervals of 4 years (2011-2014 and 2015-2018) for better data presentation.

\section{Test-episode and patient-episode definitions}

Laboratory analysis was performed using an antigen ELISA or by PCR. Some laboratories use the GeneXpert Rapid Point of Care Test, which is based on PCR and is performed in the laboratory. Individuals were uniquely identified by their CPR number. We excluded tests with inconclusive results as well as individuals with invalid or temporary CPR numbers, which is frequently given to tourists. Many individuals had several tests performed within a short time. To address multiple episodes of testing in the same patient, we considered tests performed within a 30-day time period as relating to the same episode of illness. Thus, we defined a test-episode as the series of one or more tests done on the same individual within a 30 -day period starting on the date of the first sample. Test-episodes were either positive or negative based on the results of tests: one or more positive tests in a test-episode defined a patientepisode, while a series of only negative tests defined a negative test-episode. To assess the sensitivity of the 30-day time window, we also explored 60-days and 1-year time windows.

\section{Data analysis}

Descriptive analyses in terms of time, place and person were performed. The number of test-episodes, patient-episodes and incidences hereof were calculated by sex, age group and region. We counted the proportion of positive and negative test-episodes in addition to the test positivity rate. We assessed the significance of the increasing trends with the Cuzick test. We described and determined the duration of each season between 2011/2012 and $2017 / 2018$. We used time series analysis and calendar plots to explore disease count distribution by week. Data between 2011 and 2018 were modelled by using Poisson regression, adjusted for seasonality using sine and cosine functions. The observed seasons were roughly classified as large (L) and small (S) according to their size related to the Poisson model. All calculations were performed by using STATA version 14 (StataCorp, Texas, USA).

\section{Laboratory practice survey}

To describe testing practices, a questionnaire was sent in May 2018 by email to representatives from all KMAs. The questionnaire included open and closed questions about the methodology and recommendations used in each laboratory during the past 7 years (2011-2017). We asked KMA representatives about how the test for NoV was performed (individually or as a part of packages for multiple pathogens) and about the methodology used in the laboratories/hospitals (RT-PCR, ELISA or the GeneXpert). Participants were also asked about any changes in the methods used during the study period. Finally, we asked if laboratories followed recommendations regarding the number of samples analysed per individual, the number of patients tested per outbreak group or regarding test of individuals from risk groups (age group, sex, comorbidity or immunosuppression).

\section{Results}

\section{Descriptive analysis: age, sex, place, and test performance}

Between 2011 and 2018, a total of 177397 laboratory analyses for NoV could be found and extracted from MiBa, of which 19955 (11\%) were positive. They constituted 142648 test-episodes (series of tests on the same individual) of which 15809 (11\%) were positive, representing patient-episodes. The number of tests within the test-episodes varied, $83 \%$ of patients had one sample analysed, $12 \%$ had two, $5 \%$ had three or more (max. 15 samples). In total, 24449 test-episodes consisted of two tests performed on the same individual. In $4 \%$ of these, the test results were diverging. In total, 7658 test-episodes consisted of three or more tests. In total, $1 \%$ had diverging results. Defining episodes by 60 -days and 365-days' time windows, these results changed only marginally. With the 60-days window there were 139071 test-episodes of which 15688 were positive (11\%) and with a 365-days window there were 129828 test-episodes of which 15450 were positive (12\%). The following results are therefore based on a 30 -days window. Table 1 shows the distribution of the number of tests, test-episodes, tests result and mean population size per region during two different periods. The total number of tests doubled from 
Table 1. Number of NoV tests, positive tests, test-episodes, patient-episodes, population and incidence per person-year in two time periods using a 30 days window between test series

\begin{tabular}{|c|c|c|c|c|c|c|c|c|c|}
\hline Region & $\begin{array}{l}\text { Population } \\
\text { (average) }\end{array}$ & Tests & $\begin{array}{l}\text { Positive } \\
\text { tests }\end{array}$ & $\begin{array}{l}\text { Test positive } \\
\text { rate }\end{array}$ & Test-episodes & Patient-episodes & $\begin{array}{l}\text { Test-episodes } \\
\text { positive rate }\end{array}$ & $\begin{array}{l}\text { Test-episodes } \\
\text { incidence }^{a}\end{array}$ & $\begin{array}{l}\text { Patient-episodes } \\
\text { incidence }^{a}\end{array}$ \\
\hline \multicolumn{10}{|l|}{ 2011-2014 } \\
\hline Northern & 580436 & 3966 & 596 & 15.0 & 3103 & 504 & 16.2 & 134 & 22 \\
\hline Central & 1269757 & 66 & 23 & 34.8 & 56 & 19 & 33.9 & 1 & 0 \\
\hline Southern & 1201558 & 15601 & 2407 & 15.4 & 12798 & 1932 & 15.1 & 266 & 40 \\
\hline Zealand & 817443 & 2403 & 410 & 17.1 & 2217 & 361 & 16.3 & 68 & 11 \\
\hline Capital & 1726199 & 40669 & 5652 & 13.9 & 27138 & 3875 & 14.3 & 393 & 56 \\
\hline Total & 5595394 & 62705 & 9088 & 14.5 & 45312 & 6691 & 14.8 & 202 & 30 \\
\hline \multicolumn{10}{|l|}{ 2015-2018 } \\
\hline Northern & 587828 & 3867 & 641 & 16.6 & 3625 & 546 & 15.1 & 154 & 23 \\
\hline Central & 1307024 & 2763 & 627 & 22.7 & 2477 & 551 & 22.2 & 47 & 11 \\
\hline Southern & 1217744 & 19009 & 2212 & 11.6 & 15412 & 1712 & 11.1 & 316 & 35 \\
\hline Zealand & 832279 & 14626 & 1181 & 8.1 & 13511 & 1104 & 8.2 & 406 & 33 \\
\hline Capital & 1812458 & 74427 & 6206 & 8.3 & 62311 & 5205 & 8.4 & 859 & 72 \\
\hline Total & 5757333 & 114692 & 10867 & 9.5 & 97336 & 9118 & 9.4 & 423 & 40 \\
\hline \multicolumn{10}{|l|}{ 2011-2018 } \\
\hline Northern & 584397 & 7833 & 1237 & 15.8 & 6728 & 1050 & 15.6 & 144 & 22 \\
\hline Central & 1290472 & 2829 & 650 & 23.0 & 2533 & 570 & 22.5 & 25 & 6 \\
\hline Southern & 1210109 & 34610 & 4619 & 13.3 & 28210 & 3644 & 12.9 & 291 & 38 \\
\hline Zealand & 824994 & 17029 & 1591 & 9.3 & 15728 & 1465 & 9.3 & 238 & 22 \\
\hline Capital & 1775960 & 115096 & 11858 & 10.3 & 89449 & 9080 & 10.2 & 630 & 64 \\
\hline Total & 5685931 & 177397 & 19955 & 11.2 & 142648 & 15809 & 11.1 & 314 & 35 \\
\hline
\end{tabular}

Incidence per 100000 person-year. 
Fig. 1. NoV patient-episode incidence by sex and age group and overall proportion of patient-episodes and test-episodes (positivity rate) by age group, Denmark 2011-2018.
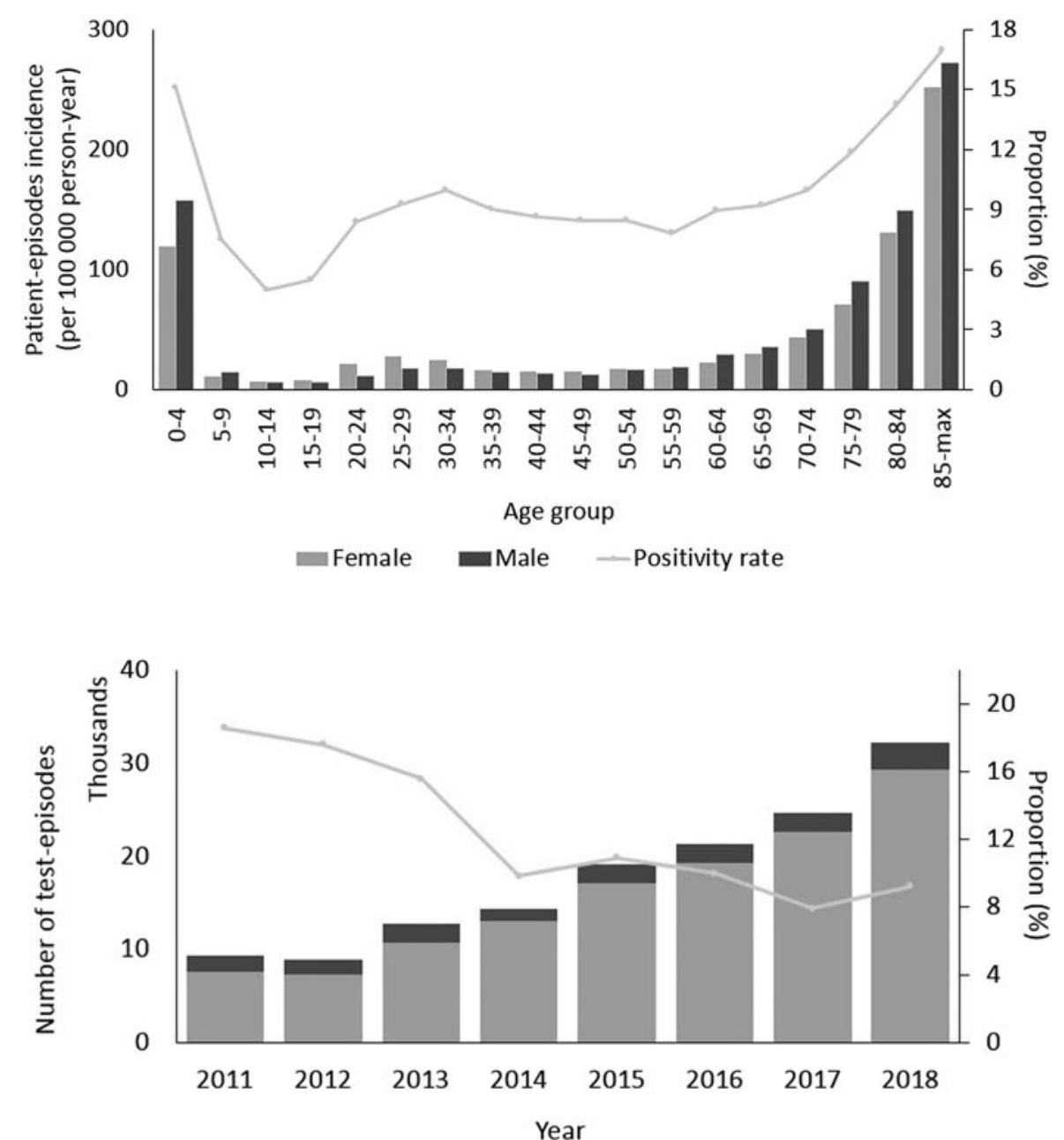

Fig. 2. Number of test episodes, patient-episodes and negative test-episodes per year and proportion of patient-episodes and test-episodes (positivity rate), Denmark 2011-2018.

Negative-episodes $\square$ Patient-episodes - Positivity rate
62705 during the first period to 114692 in the second period whereas the positivity rate decreased from $14.5 \%$ to $9.5 \%$. Most tests were done in the Capital Region (65\% of all tests) and the least in the Central Region (2\%). During 2015-2018, the Capital Region performed 859 tests per 100000 population whereas the Central Region performed 47 tests per 100000 population.

The highest proportion of positive test episodes were in patients less than 5 years old (15\%) or older than 85 years (17\%) (Fig. 1). There was a noticeable increase in incidence among older age groups (60 and more years old, $P<0.05$ ). Incidence was larger among females than males in the age group between 20 and 34 years. In contrast, among the elderly above 60 years of age incidence was larger among males than females. The largest difference in sex incidence was in males (157 per 100000 person-years) compared to females (119) in the youngest age group (Fig. 1). During the study period, due to an increasing trend in the number of test-episodes performed (from 9366 in 2011 to 32260 in 2018, P $<0.05$ ) (Fig. 2), the positivity rate decreased as the number of positive test-episodes only increased slightly by year.

\section{Laboratory practise survey}

Nine out of 10 present laboratories answered the survey. Existing $\mathrm{NoV}$ data showed that $89 \%$ of laboratory results were obtained using RT-PCR to detect virus RNA. During the study period, some laboratories introduced in-house RT-PCRs. Methods used in the individual laboratories as well as introduced changes including approximate dates are summarised in Table 2. The introduction of RT-PCR in some laboratories in Zealand and Central Regions resulted in a slight increase in the number of tests performed. The regional laboratory in the Northern Region introduced RT-PCR in 2013 that surprisingly did not result in an increase as was observed in the other laboratories. However, in 2015, after the introduction of the GeneXpert test method, the number of tests almost doubled. There was a concurrent decrease in test numbers performed at the reference laboratory, which is placed at SSI, in the Capital Region because this laboratory has mainly been diagnosing $\mathrm{NoV}$ in cases related to suspicions of foodborne outbreaks from 2015 onwards. In January 2016, one of the Capital Region laboratories, placed in the tertiary hospital 'Rigshospitalet' included NoV analyses in a 'package' to diagnose gastroenteritis, also including tests for other viruses and bacteria. As a result, the number of reported tests doubled in the following years.

Recommendations of the laboratories in connection with nosocomial outbreaks are not uniform. For example, some of the hospitals (in Zealand and Capital Regions) sample only the first patient during outbreaks, whereas other hospitals 
Table 2. Number of NoV tests in different regions with comment about main changes in testing methods in regional laboratories, Denmark 2011-2018

\begin{tabular}{|c|c|c|c|c|c|c|c|c|c|c|}
\hline \multirow[b]{2}{*}{ Region } & \multirow{2}{*}{$\begin{array}{l}\text { No. of KMAs active } \\
\text { during study } \\
\text { period }\end{array}$} & \multicolumn{8}{|c|}{ Year } & \multirow[b]{2}{*}{ Comment } \\
\hline & & 2011 & 2012 & 2013 & 2014 & 2015 & 2016 & 2017 & 2018 & \\
\hline Northern & 1 & 1102 & 1208 & 985 & 671 & 1173 & 719 & 822 & 1153 & $\begin{array}{l}\text { January } 2010 \text { - Norovirus antigen test (EIA Test from } \\
\text { Oxoid); } \\
\text { July } 2013 \text { - Introduction of RT-PCR (Altona } \\
\text { Diagnostics); January } 2015 \text { - Change to GeneXpert (Xpert } \\
\text { Norovirus, Cepheid) }\end{array}$ \\
\hline Central & 2 & 0 & 0 & 0 & 66 & 522 & 707 & 580 & 954 & $\begin{array}{l}\text { October } 2014 \text { - Introduction of in-house RT-PCR; } \\
\text { March } 2015 \text { - Expanding testing method for whole region }\end{array}$ \\
\hline Southern & 3 & 3351 & 4068 & 4154 & 4028 & 4680 & 4012 & 4463 & 5854 & $\begin{array}{l}\text { Before } 2013 \text { - Use of individual test and package for viral } \\
\text { diseases (NoV + rotavirus); } \\
\text { After } 2013 \text { - Introduction of PCR package for viral } \\
\text { diseases (NoV + rotavirus + adenovirus + sapovirus + } \\
\text { astrovirus); } \\
\text { January } 2018 \text { - Introduction of GeneXpert }\end{array}$ \\
\hline Zealand & 1 & 0 & 115 & 749 & 1539 & 2456 & 2977 & 3716 & 5477 & September 2012 - Introduction of in-house RT-PCR \\
\hline Capital & 4 & 4312 & 3855 & 9130 & 10264 & 13556 & 15417 & 19150 & 24241 & $\begin{array}{l}2013 \text { - Introduction of in-house RT-PCR in one } \\
\text { laboratory; } \\
2010-2016 \text { - Use of package for viral diseases (NoV + } \\
\text { rotavirus + adenovirus) by RT-PCR (BD-max); } \\
\text { January } 2016 \text { - Change to gastroenteritis package (virus } \\
\text { + bacteria) by RT-PCR (C-gene) }\end{array}$ \\
\hline $\begin{array}{l}\text { Reference } \\
\text { laboratory }\end{array}$ & 1 & 6256 & 4494 & 1384 & 974 & 517 & 497 & 401 & 648 & $\begin{array}{l}\text { January } 2015 \text { - Diagnostic performed mainly due to } \\
\text { foodborne outbreaks }\end{array}$ \\
\hline Total & 12 & 15021 & 13740 & 16402 & 17542 & 22904 & 24329 & 29132 & 38327 & \\
\hline
\end{tabular}


(Southern and Capital Regions) reported to be testing all patients affected by an outbreak. In addition, varying instructions were identified regarding the recommended number of samples to be tested: most laboratories recommended taking 1-2 samples per patient whereas a few hospitals in the Capital Region recommended three samples per patient.

\section{Seasonal variation}

Figure 3 shows the weekly distribution of the NoV patientepisodes. There was a very clear seasonal variation in NoV infection with the majority of patient-episodes occurring in the winter months (December-February), peaking around weeks $4-5$. The Poisson regression model fitted to the seasonal data highlights how the patient-episodes increase at a similar time each year, around week 45 (November) and decrease in week 14 (March). The seasonal pattern of the NoV patient-episodes seems to change biannually, while the number of patient-episodes seems to rotate between 'large' and 'small' seasons. Correspondingly, seasons from 2011 to 2018 can be classified as 2011/2012 (small), 2012/2013 (large), 2013/2014 (small), 2014/2015 (large), 2015/ 2016 (small), 2016/2017 (small) and 2017/2018 (large). Seasons $2015 / 2016$ and $2016 / 2017$ were atypical in their shape: longer by 2-3 weeks and less intensive in comparison with other seasons. The last season, 2017/2018 was more intensive with the highest number of patient-episodes (Fig. 3).

\section{Discussion}

For the first time in Denmark, laboratory-based data on diagnosed NoV tests were compiled and described in terms of time, place and person. Over the study period, the number of $\mathrm{NoV}$ tests increased annually. Test frequency varied highly between different hospitals in Denmark. The majority of tests were performed in children and elderly patients. These groups also had the highest proportions of positive tests, suggesting that the real incidence of disease is higher in these age groups or alternatively it might reflect the hospitalisation age group incidence. There was a marked seasonal variation, with the season running from the end of December to February.

The epidemiological patterns are similar to what has been reported from other countries. Even though NoV is associated with acute gastroenteritis among children, recent studies show that other age groups such as the elderly are also at high risk if living in institutional settings. NoV outbreaks are common in hospitals or nursing homes [9, 25, 26]. In Denmark, data on healthcare settings outbreaks are not collected. However, if national laboratory data could be linked to the national hospitalisation database, this could in theory allow us to monitor the nosocomial outbreaks. Such information could be of value to hospitals, working on reducing the impact of NoV outbreaks.

The increasing test activity in Denmark could be explained by the gradual introduction of RT-PCR and the fact that more tests are performed as a part of multi-pathogen testing for gastroenteritis. Laboratory identification of $\mathrm{NoV}$ is useful for guidance of patient isolation measures as well as the overall reduction of NoV transmission through infection control and public health interventions [27, 28]. Currently in Denmark, nine of 10 KMAs are using an RT-PCR to diagnose NoV. In the near future the rapid point-of-care test popularity may increase, which also can play a role in increasing the number of tests. However, if such

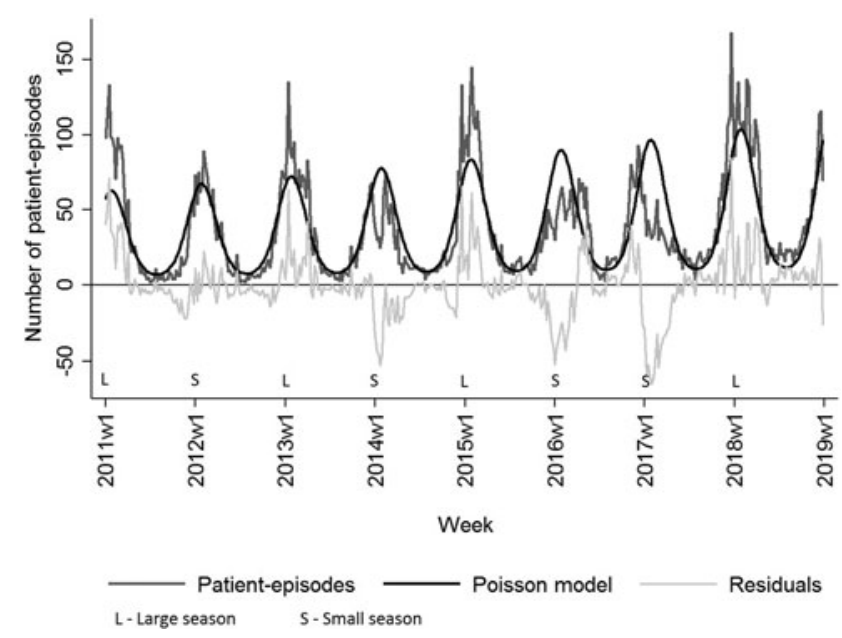

Fig. 3. Number of weekly NoV laboratory-diagnosed patient-episodes, number of modelled patient-episodes and residuals hereof, Denmark 2011-2018. NoV seasons are classified as larger than modelled or smaller than modelled.

testing is introduced as bedside testing, these cases might escape laboratory-based surveillance.

We saw a strong seasonal distribution. In Denmark, foodborne NoV outbreaks are reported throughout the year, however, the known foodborne outbreak cases constituted only a small fraction of the cases in our dataset. A marked seasonal distribution with a peak in the colder months of cases, and to a lesser extent of outbreaks, was described in a recent analysis of published papers [29]. It also showed that northern European countries generally report that most cases occur in December-February. The seasonal patterns may also be affected if the viruses in circulation are changing $[1,2,12]$. The NoV genome has a high mutation rate and NoV may undergo genetic shifts which result in new strains that are able to infect partially immune hosts that already experienced the old strains. A review of NoV strains circulating over the last decades showed emergent strains replacing previous ones, resulting in new global epidemics, appearing every 2-4 years [26]. The time and scale of NoV seasonal activity in Denmark differs from season to season. One explanation of this pattern could be that immunity in the population, after a large season, has increased resulting in fewer cases during the next season. Changes in the dominating genotype render the population renewed susceptible. In late 2014, researchers from Asia reported a major shift from the predominant GII.4 to the novel GII.P17-GII.17 NoV strain (GII.17 Kawasaki 2014). The novel GII.P17-GII.17 NoV variant circulated widely in Europe in the season 2015-2016 [30, 31]. In Denmark, the new GII.17 Kawasaki 2014 strain was identified for the first time in late 2015 and first identified in connection with a foodborne outbreak early in 2016 [32], which supports the theory of the biennial changes. In many developed countries, as well as in Denmark, outbreaks in healthcare settings are most often caused by NoV genogroup II genotype 4 (GII.4). This genotype is mostly associated with the elderly population $[33,34]$. The absence of routine surveillance on the gastroenteritis outbreaks in hospitals limits the information about changes in virus variants circulating in healthcare settings.

Although this study covers data available in the national $\mathrm{MiBa}$ database from 2011 through 2018, we acknowledge the possibility that not all diagnosed NoV cases were identified. Timely monitoring of the cases could help identify the beginning of the season, which would likely improve the prevention and control measures 
by public health and healthcare settings personnel, for instance help with planning for increased healthcare usage in facilities or alert messages to the public. It would be important to further investigate the NoV seasonal activity after passive surveillance system implementation. A passive surveillance system for $\mathrm{NoV}$ in Denmark is anticipated to be approved within the near future. This automated data collection should provide timely and relevant information for public health action; in the future, if combined with for instance the hospital discharge database, it might assist in identifying and managing further outbreaks.

In conclusion, this study suggests that the introduction of an automated passive NoV laboratory-based surveillance system in Denmark would be helpful both for long-term trend analyses and for managing outbreaks within hospitals and healthcare facilities. If information on hospitalisation status could be included in the surveillance, it would be a valuable tool in the managing of hospital outbreaks and the evaluation of their impact. Likewise, timely identification of the beginning of and the strength of an annual NoV season could provide a preparedness signal to alert healthcare facilities. This could guide implementation of control measures and thus aid in reducing the substantial morbidity as well as the economic impact of NoV outbreaks.

Acknowledgements. The authors thank all Departments of Clinical Microbiology of all Danish hospitals for providing information about the diagnostic methods used in the laboratories.

Financial support. This research did not receive any specific grant from funding agencies in the public, commercial or not-for-profit sectors.

Conflict of interest. None.

\section{References}

1. Inns T and Harris J (2017) Community-based surveillance of NoV disease: a systematic review. BMC Infectious Diseases 17, 657.

2. Ahmed S et al. (2014) Global prevalence of NoV in cases of gastroenteritis: a systematic review and meta-analysis, Lancet Infectious Diseases 14, 725-730. Published online: 27 June 2014.

3. Van Asten L et al. (2011) Unspecified gastroenteritis illness and deaths in the elderly associated with norovirus epidemics. Epidemiology (Cambridge, Mass.) 22, 336-343.

4. Harris JP et al. (2008) Deaths from norovirus among the elderly, England and Wales. Emerging Infectious Diseases 14, 1546-1552.

5. Wolffs PF et al. (2011) Replacing traditional diagnostics of fecal viral pathogens by a comprehensive panel of real-time PCRs. Journal of Clinical Microbiology 49, 1926-1931.

6. Bobo LD and Dubberke ER (2010) Recognition and prevention of hospital-associated enteric infections in the intensive care unit. Critical Care Medicine 38(suppl. 8), S324-S334.

7. Fraizel HA et al. (2014) Identifying Sources and Transmission Routes of Norovirus Outbreaks (dissertation). Erasmus University in Roterdam, Netherlands, ISBN/EAN: 978-90-6464-762-8.

8. Brandhof WE et al. (2004) Costs of gastroenteritis in The Netherlands. Epidemiology and Infections 132, 211-221.

9. Lindsay L et al. (2015) A decade of norovirus disease risk among older adults in upper-middle- and high-income countries: a systematic review. BMC Infectious diseases 15, 425 .

10. Patel MM et al. (2008) Noroviruses: a comprehensive review. Journal of Clinical Virology 44, 1-8. Published online: 11 December 2008.

11. Ettayebi K et al. (2016) Replication of human noroviruses in stem cellderived human enteroids. Science (New York, N.Y.) 353, 1387-1393.
12. Centre for Disease Control and Prevention (2011) Updated norovirus outbreak management and disease prevention guidelines. Morbidity and Mortality Weekly Report 60, 3.

13. Harris J (2016) Norovirus surveillance: an epidemiological perspective. The Journal of Infectious Diseases 213, S8-S11. Published online: 01 February 2016.

14. Lopman B et al. (2009) Host, weather and virological factors drive norovirus epidemiology: time-series analysis of laboratory surveillance data in England and Wales. PLoS One 4, e6671.

15. Pires SM et al. (2019) Burden of disease estimates of seven pathogens commonly transmitted through foods in Denmark, 2017. Foodborne Pathogens and Disease doi: 10.1089/fpd.2019.2705.

16. Müller L et al. (2015) Separate norovirus outbreaks linked to one source of imported frozen raspberries by molecular analysis, Denmark, 20102011. Epidemiology and Infections 143, 2299-2307.

17. Franck KT et al. (2014) Norovirus epidemiology and patient age, Denmark. Emerging Infectious Diseases 20. Available at www.cdc.gov/ eid, http://dx.doi.og/10.32301/eid2007.130781.

18. Müller L et al. (2016) Series of norovirus outbreaks caused by consumption of green coral lettuce, Denmark, April 2016. PLoS Currents 8. doi: https://www.ncbi.nlm.nih.gov/pmc/articles/PMC5074700/.

19. Franck KT et al. (2015) Sources of calicivirus contamination in foodborne outbreaks in Denmark, 2005-2011 - the role of the asymptomatic food handler. The Journal of Infectious Diseases 211, 563-570.

20. Anon (2017) Annual Report on Zoonoses in Denmark 2016, National Food Institute, Technical University of Denmark.

21. Anon (2019) Annual Report on Zoonoses in Denmark 2018, National food Institute, Technical University of Denmark.

22. Voldstedlund $M$, Haarh $M$ and Mølbak $K$ and The MiBa Board of Representatives (2014) The Danish Microbiology Database (MiBa) 2010 to 2013. EuroSurveillance 19, 20667.

23. Pedersen CB (2011) The Danish civil registration system. Scandinavian Journal of Public Health 39(suppl. 7), 22-25. http://dx.doi.org/10.1177/ 1403494810387965.

24. Danmarks statistic. StatBank database. Available at https://www.dst.dk/en (Accessed 19 July 2019).

25. Corcoran MS, Van Well GT and Van Loo IH (2014) Diagnosis of viral gastroenteritis in children: interpretation of real-time PCR results and relation to clinical symptoms. European Journal of Clinical Microbiology \& Infectious Diseases 33, 1663-1673. Published online: 16 May 2014.

26. Glass RI et al. (2009) Norovirus gastroenteritis. The New England Journal of Medicine 361, 1776-1785. doi: 10.1056/NEJMra0804575.

27. Vinje $\mathbf{J}$ (2015) Advances in laboratory methods for detection and typing of norovirus. Journal of Clinical Microbiology 53, 373-381. Published online: 29 October 2009.

28. Rooney BL et al. (2014) Detection of circulating norovirus genotypes: hitting a moving target. Virology Journal 11, 129.

29. Ahmed SM et al. (2013) A systematic review and meta-analysis of the global seasonality of norovirus. PLoS ONE 8(10). doi: https://doi.org/10. 1371/journal.pone.0075922.

30. Van Beek J et al. (2018) Molecular surveillance of norovirus, 2005-2016: an epidemiological analysis of data collected from Noronet network. Lancet Infectious Diseases 18, 545-553. Published online: 26 January 2018.

31. Chan MCW et al. (2017) Global spread of norovirus GII.17 Kawasaki 308, 2014-2016. Emerging Infectious Diseases 23, 8.

32. Dam Rasmussen L et al. (2016) Molecular evidence of oysters as vehicle of norovirus GII.P17-GII.17. Emerging Infectious Diseases 22, 2024.

33. Frank KT et al. (2014) Norovirus epidemiology in community and health care settings and association with patient age, Denmark. Emerging Infectious Diseases 20, 1123-1131.

34. Frank KT et al. (2015) Norovirus genotypes in hospital settings: differences between nosocomial and community-acquired infections. The Journal of Infectious Diseases 212, 881-888. Published online: 15 September 2015 . 